\title{
Relationship between fish-eating habits, cytokine production and chronic urticaria, with a special emphasis on Anisakis simplex associated sensitization associated chronic urticarial
}

\author{
A. Daschner ${ }^{1}$, A. Valls ${ }^{1}$, C. de Frutos ${ }^{1}$, V. Fernández-Fígares ${ }^{2}$, M. Rodero ${ }^{2}$ and C. Cuéllar ${ }^{2}$ \\ ${ }^{1}$ Servicio de Alergia, Instituto de investigación Sanitaria-Hospital Universitario de la Princesa, Madrid, Spain and \\ ${ }^{2}$ Departamento de Parasitología, Facultad de Farmacia, Universidad Complutense, Madrid, Spain
}

\begin{abstract}
A phenotype of chronic urticaria has been associated with sensitization against the fish-parasite Anisakis simplex and could have a different prognosis. Fish eating habits are associated with sensitization against A. simplex in gastro-allergic Anisakiasis (acute allergic reaction) as well as $\mathrm{CU}+$, but could also be associated with the prognosis. We therefore sought to analyse the relationship between fisheating habits and cytokine production in patients with $\mathrm{CU}+$ compared with non-sensitized chronic urticaria (CU-) patients. Chronic urticaria patients were included with at least twice a week occurring wheals for at least 6 weeks. Patients were included in the CU + if they displayed a positive skin prick test and detectable serum IgE against A. simplex. CU- patients did not have positive SPT or specific IgE. A standardized questionnaire delivered information about weekly oily, total and canned fish and monthly portions of anchovies in vinegar sauce. Supernatants of PBMCs stimulation assays were used for measurement of cytokine production (IL-2, IL-4, IL-6, IL-10, TNF- $\alpha$, IFN- $\gamma$ and IL-17) after cell culture stimulated with A. simplex crude extract or Concanavalin A. Bivariate analysis, as well as multivariate analysis (regression model) were used to search for the relationship between the urticaria phenotype, fish-eating habits and cytokine production. Total fish intake was associated with $\mathrm{CU}+$, whereas oily fish intake was associated with $\mathrm{CU}$. In patients with $\mathrm{CU}+$, total fish intake was correlated with Con $A$ induced IL-6 and IL-17 (Rho $>0.5 ; p<0.05$ ). In the CU- group total fish intake was associated with $A$. simplex induced IL-2 (Rho $0.46 ; p<0.05$ ), but negatively correlated with $A$. simplex induced TNF- $\alpha($ Rho $-0.52 ; p=0.02)$. Oily fish intake was also associated with A. simplex induced IL-2 (Rho $0.56 ; p=0.01$ ). Anchovies in vinegar sauce intake was negatively associated with TNF- $\alpha$ and IFN- $\gamma($ Rho $<-0.53 ; p<0.02)$. Canned fish was negatively correlated with IL-4 production (Rho 0.44 ; $p=0.05)$. In conclusion, even in non-sensitized patients, the dissociation of IL-2 and TNF- $\alpha$ production after A. simplex stimulation favours the notion that $A$. simplex derived products present frequently in fish modulate immunologic markers. Higher pro-inflammatory IL-6 and IL-17 production associated with a higher total fish intake seems to be counter intuitive to the fact that fish oils have previously been shown to have anti-inflammatory properties. Therefore, the special situation of chronic urticaria could be responsible. Further studies could use biomarkers of fish ingestion to confirm these findings and consider possible dietary intervention.
\end{abstract}

